\title{
Time-Discrete Geodesics in the Space of Shells
}

\author{
B. Heeren ${ }^{1}$, M. Rumpf ${ }^{1}$, M. Wardetzky ${ }^{2}$ and B. Wirth ${ }^{3}$ \\ ${ }^{1}$ Institute for Numerical Simulation, University of Bonn, Germany \\ ${ }^{2}$ Institute of Num. and Appl. Math, University of Göttingen, Germany \\ ${ }^{3}$ Courant Institute, New York University, USA
}
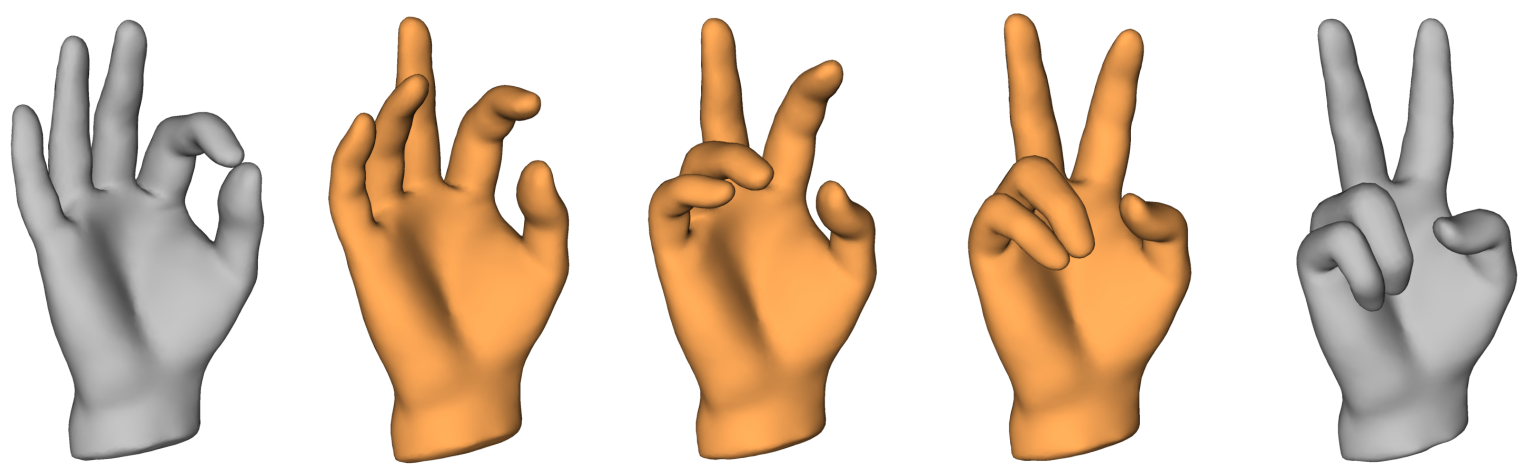

Figure 1: Discrete geodesic computed from two input poses (leftmost and rightmost hand).

\begin{abstract}
Building on concepts from continuum mechanics, we offer a computational model for geodesics in the space of thin shells, with a metric that reflects viscous dissipation required to physically deform a thin shell. Different from previous work, we incorporate bending contributions into our deformation energy on top of membrane distortion terms in order to obtain a physically sound notion of distance between shells, which does not require additional smoothing. Our bending energy formulation depends on the so-called relative Weingarten map, for which we provide a discrete analogue based on principles of discrete differential geometry. Our computational results emphasize the strong impact of physical parameters on the evolution of a shell shape along a geodesic path.
\end{abstract}

Categories and Subject Descriptors (according to ACM CCS): I.3.5 [Computer Graphics]: Computational geometry and object modeling_-Physically based modeling

\section{Introduction}

Imagine being presented with a picture of two different poses of a shape, such as two postures of a human hand or an animal body. Based on everyday experience, it might be obvious how to deform one such shape into the other with the least amount of effort or in a most natural manner. Nonetheless, irrespective of how well the human brain might be capable of reconstructing such deformations, the question of how to do so automatically remains a challenge.

We offer a step toward an automatic construction of deformation paths between two-dimensional surfaces, based on a physical deformation paradigm. Viewing surfaces as thin viscous materials of some finite (albeit small) thickness, we define the best deformation path between two points in the space of shells as one that has least energy dissipation, i.e., that requires the least total effort (cf. Fig. 1). Although there might be countless ways to equip shell space with a notion of distance in principle, our physically motivated approach appears to conform with human intuition. E.g., geodesic paths corresponding to our distance notion tend to automatically prevent undesired singularities, such as the formation of creases or a local collapse of the surface. Our geometric 
formulation is invariant under rigid motions and does not require any surface parameterization. We introduce the notion of time-discrete geodesics, which provides a computationally efficient and robust time discretization of geodesics in shell space, yielding intermediate shapes that are stable with respect to the spatial discretization of input poses.

The requisite viscous dissipation potential of our model is closely related to elastic properties of thin shells. We rely on recent mathematical results that offer a sound justification for (tangential) membrane and (out-of-plane) bending contributions of smooth thin shells as their thickness approaches zero. In particular, we employ the so-called relative Weingarten map for describing bending energy contributions, and we offer a discrete theory that closely parallels the smooth one.

We demonstrate how appropriate choices of material parameters in our model allow for controlling the appearance of geodesic paths, resulting in an intuitive paradigm for navigating within shell space.

\section{Related work}

There exists a vast amount of literature on interpolating and morphing shapes. One possible approach linearly interpolates local geometric quantities such as edge lengths and dihedral angles of triangulated surfaces and then seeks the triangulated surface whose edge lengths and dihedral angles fit best to the interpolated values, either by optimal alignment and blending of patches [WDAH10] or by minimizing the total edge length and dihedral angle difference to the interpolated values [FB11].

Sometimes, morphing paths are computed as the gradient flow of an energy that measures the mismatch between a template and a reference shape [CFK05]. By designing an appropriate inner product with respect to which the gradient is defined, paths with certain properties (such as smoothness or volume preservation) tend to be preferred [EPT*07].

An alternative approach is to design energies that yield morphing paths with a specifically desired behavior $\left[\mathrm{AOW}^{*} 08\right]$. Such an approach is conceptually close to the more general setting of Riemannian shape spaces-an approach that we also follow in this paper. The respective Riemannian spaces have been considered for volumetric shape deformations, where the metric imitates a physical energy dissipation induced by the deformation of a ductile or viscous material. This dissipation is either measured within shapes [FJSY09, FW06] or in the surrounding space [DGM98]. Such physical approaches tend to yield intuitive paths and allow for a simple and natural time discretization [WBRS11]. Our approach is inspired by this idea.

For planar curves, different Riemannian metrics have been devised, including curvature-weighted $L^{2}$ - or Sobolevtype metrics [MM06, SYM07]. Such metrics can sometimes be interpreted as a measure of stretching and bending variations [SKJJ11, SMSY11]. In contrast to volumetric approaches, Riemannian metrics for spaces of curves or surfaces in particular benefit from the additional bending term that regularizes curvature changes - an observation that we also exploit in this paper. With regards to the space of surfaces, $H^{1}$-type metrics on surface variations have been studied [BB11], as well as $L^{2}$-metrics on special representations of parameterized surfaces [KKG* $11, \mathrm{KKDS} 10]$. As opposed to physics-based approaches, parameterization-based metrics are not intrinsically blind to rigid body motions. Moreover, their induced distance is related to the extrinsic difference between surfaces in ambient space, so that even isometric surfaces might be far apart from one another. A combination of extrinsic and intrinsic distance is presented in [BBK09].

In a different approach, Kilian et al. consider geodesics between consistently triangulated surfaces [KMP07], where the Riemannian metric measures stretching of triangle edges. Their metric is intrinsically invariant with respect to isometric deformations; however, in order to select between different isometric paths, a supplementary $L^{2}$-regularization is required. The metric by Liu et al. also takes into account directional changes of edges [LSDM10]. Although the latter examples explore the space of surfaces, they do not offer a physically motivated model for the underlying structures.

Our physics-based approach builds on existing literature for discretizing thin shells in a geometric manner and on concepts known from Discrete Exterior Calculus (DEC) [DHLM05, DKT08]. We adapt ideas from Grinspun et al., who derive a simple shell model for triangular meshes, consisting of a membrane and a bending term, where the latter captures differences in mean curvature [GHDS03]. In particular, we build on the extension to shape operators provided in [GGRZ06].

From a mathematical perspective, in devising our Riemannian shell space, we rely on recent analytical results for smooth surfaces, where a curvature-based deformation energy of thin plates is rigorously derived as a socalled $\Gamma$-limit of three-dimensional nonlinear elasticity theory [FJM01, FJM02] and extended to shells in [FJMM03].

\section{A discrete, physical path energy}

Consider the question of finding the "best" deformation path between two given thin shells, i.e., between two thin, curved layers of material that can undergo smooth deformations in $\mathbb{R}^{3}$. In this section, we offer a physically motivated answer to this question, based on paths of least energy dissipation; we later cast this answer into a more geometric picture.

Time-continuous setting. Viewing shells as thin threedimensional materials, let $\delta$ be a scale parameter that reflects the thickness of a smooth surface $\mathcal{M}$ embedded into 
$\mathbb{R}^{3}$, and let $\mathcal{M}^{\delta}$ denote the corresponding material around $\mathcal{M}$. Consider now a family $\left(\phi_{t}\right)_{0 \leq t \leq 1}$ of diffeomorphisms with $\phi_{0}=$ Id. Then $\phi_{t}$ generates a deformation path $\phi_{t}\left(\mathcal{M}^{\delta}\right)$. Suppose that shells were made of a viscous material, then such a deformation would lead to viscous friction within the shell's volume. The density of viscous friction-known as dissipation-can be described as a quadratic function $\operatorname{Diss}\left(\varepsilon\left[v_{t}\right]\right)$ of the symmetrized gradient

$$
\varepsilon\left[v_{t}\right]:=\frac{1}{2}\left(\left(\mathrm{D} v_{t}\right)^{T}+\mathrm{D} v_{t}\right)
$$

of the corresponding Eulerian flow field $v_{t}=\dot{\phi}_{t} \circ \phi_{t}^{-1}$. We treat viscous dissipation according to Rayleigh's analogy that derives a viscous formulation from an elastic one by replacing elastic strain by strain rates [Str45].

To this end, we consider an elastic energy density $W(A)$ acting on symmetric $3 \times 3$ matrices (strain tensors) $A$. In order to maintain invariance under rigid motions, we assume that $W\left(R^{T} A R\right)=W(A)$ for all $R \in S O(3)$. We further assume that the identity matrix, $\mathbb{1}$, is a minimizer, implying that $W(\mathbb{1})=0$ and $W_{A}(\mathbb{1})=0$, where $W_{. A}(\mathbb{1})$ denotes the derivative of $W$ at the identity. According to Rayleigh's analogy, we consider the dissipation density to be given by the second derivative of elastic energy density at the identity, i.e.,

$$
\operatorname{Diss}(\delta A)=\frac{1}{2} W_{, A A}(\mathbb{1})(\delta A, \delta A)
$$

for any symmetric $3 \times 3$ matrix $\delta A$. Here $W_{, A A}(\mathbb{1})(\delta A, \delta A)$ denotes the second derivative of $W$ with respect to the variation $\delta A$.

The energy dissipation associated with the accumulated viscous friction along the deformation path is then given by

$$
\mathcal{E}\left[\left(\phi_{t}\right)_{0 \leq t \leq 1}\right]=\int_{0}^{1} \int_{\phi_{t}\left(\mathcal{M}^{\delta}\right)} \operatorname{Diss}\left(\varepsilon\left[v_{t}\right]\right) \mathrm{d} x \mathrm{~d} t .
$$

In this view, a best or shortest path between two shells can be defined as the path of least energy dissipation.

Time-discrete setting. In order to transform the above time-continuous setting into a corresponding time-discrete one, consider the time-discrete family $\left(\mathcal{M}_{k}^{\delta}\right)_{k=0, \ldots, K}$ of shells given by $\mathcal{M}_{k}^{\delta}=\phi_{k \tau}\left(\mathcal{M}^{\delta}\right)$, where $\tau=\frac{1}{K}$ denotes the discrete time step. According to the above discussion of Rayleigh's analogy, we define the total elastic energy by

$$
\mathcal{W}\left[\mathcal{M}^{\delta}, \phi\left(\mathcal{M}^{\delta}\right)\right]=\int_{\mathcal{M}^{\delta}} W(A[\phi]) \mathrm{d} x
$$

for a diffeomorphic deformation $\phi$ from $\mathcal{M}^{\delta}$ into $\mathbb{R}^{3}$. Here we let $A[\phi]:=\mathrm{D} \phi^{T} \mathrm{D} \phi$ specifically denote the Cauchy Green strain tensor. Using (1), a straightforward Taylor expansion shows that one can approximate, up to second order in $\tau$, the path energy (2) by

$$
\mathcal{E}\left[\mathcal{M}_{0}, \ldots, \mathcal{M}_{K}\right]:=\frac{1}{\tau} \sum_{k=1}^{K} \mathcal{W}\left[\mathcal{M}_{k-1}^{\delta}, \phi_{k}\left(\mathcal{M}_{k-1}^{\delta}\right)\right],
$$
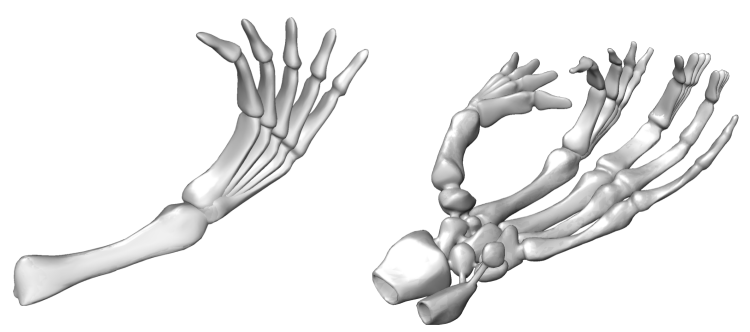

Figure 2: Two discrete geodesic paths show the (physically) optimal deformation between two poses of a finger (left) and a hand skeleton (right) (all shells are superimposed). Compare also to Fig. 5 and 6 in [KMP07].

where $\phi_{k}:=\phi_{k \tau} \circ \phi_{(k-1) \tau}^{-1}$ and $\phi_{k}\left(\mathcal{M}_{k-1}^{\delta}\right)=\mathcal{M}_{k}^{\delta}$. Notice that on the left-hand side of (4) we have tacitly represented the corresponding material volume $\mathcal{M}_{k}^{\delta}$ by the surfaces $\mathcal{M}_{k}$.

In this framework a "best discrete path" minimizes $\mathcal{E}\left[\mathcal{M}_{0}, \ldots, \mathcal{M}_{K}\right]$ under the assumption that $\mathcal{M}_{0}$ and $\mathcal{M}_{K}$ are given. Note that due to (4) we here implicitly associate a sequence $\mathcal{M}_{0}, \ldots, \mathcal{M}_{K}$ of shells with a sequence of deformations $\phi_{1}, \ldots, \phi_{K}$ of corresponding material volumes $\mathcal{M}_{0}^{\delta}, \ldots, \mathcal{M}_{K-1}^{\delta}$. This means that there is a point relation between all shells $\mathcal{M}_{k}$, and in particular the relation between $\mathcal{M}_{0}$ and $\mathcal{M}_{K}$ is given as a priori information. Also, the deformation of the material around $\mathcal{M}_{0}, \ldots, \mathcal{M}_{K}$ is simply assumed to be energetically optimal in the sense of the energy (3). Next, we provide an interpretation of this approach from a geometric point of view. Then, we approximate the energy $\mathcal{W}$ by an energy directly defined on the two-dimensional shell surface.

\section{Time-discrete geodesics in the space of shells}

Shortest geodesic paths on Riemannian manifolds are defined as minimizers of a path energy for fixed end points. Thus given a discrete path energy the definition of a discrete geodesic path suggests itself:

Definition [Discrete geodesic in the space of shells] Given two shells $\mathcal{M}_{A}$ and $\mathcal{M}_{B}$ we refer to the minimizer $\left(\mathcal{M}_{0}, \mathcal{M}_{1}, \mathcal{M}_{2}, \ldots, \mathcal{M}_{K}\right)$ of the discrete path energy (4), with $\mathcal{M}_{0}=\mathcal{M}_{A}$ and $\mathcal{M}_{K}=\mathcal{M}_{B}$, as a discrete geodesic.

As the number $K$ of shells increases and the discrete geodesic path between $\mathcal{M}_{A}$ and $\mathcal{M}_{B}$ becomes refined, one can expect convergence to a time-continuous shortest path with respect to a particular metric, which we next identify. Let $\mathcal{E}_{\tau}$ be the path energy from time 0 to time $\tau$ of a smooth path $(c(t))_{t \geq 0}$ on a Riemannian manifold with $v=\dot{c}(0)$. Then one recovers the underlying metric $g$ at $c(0)$ by $g(v, v)=\lim _{\tau \rightarrow 0} \frac{1}{\tau} \mathcal{E}_{\tau}$. Hence, in light of the approximate path energy (4) we obtain the following representation for the metric $g$ on velocity fields $v$ deforming a given shell 
(which represent the tangent vectors in shell space):

$$
g(v, v)=\lim _{\tau \rightarrow 0} \frac{1}{\tau^{2}} \mathcal{W}\left[\mathcal{M}^{\delta}, \phi_{\tau}\left(\mathcal{M}^{\delta}\right)\right]
$$

where $\left(\phi_{\tau}\right)_{\tau>0}$ is a deformation path with $\dot{\phi}_{0}\left(\mathcal{M}^{\delta}\right)=v$. For smooth deformation fields we thus retrieve the temporal rate of viscous dissipation $\int_{\mathcal{M}^{\delta}} \operatorname{Diss}(\varepsilon[v]) \mathrm{d} x$ as the associated metric. Then a simple quadrature argument shows that $\mathcal{W}\left[\mathcal{M}^{\delta}, \phi_{\tau}\left(\mathcal{M}^{\delta}\right)\right]$ is a 2 nd order approximation of the corresponding squared Riemannian distance $\operatorname{dist}^{2}\left(\mathcal{M}^{\delta}, \phi_{\tau}\left(\mathcal{M}^{\delta}\right)\right)$.

We point out an interesting fact for discrete paths on complete Riemannian manifolds that motivates the name discrete geodesic. For any discrete path (i.e., sequence of points) $\left(c_{0}, \ldots, c_{K}\right)$ one has

$$
\operatorname{dist}^{2}\left(c_{0}, c_{K}\right) \leq K \sum_{k=1}^{K} \operatorname{dist}^{2}\left(c_{k-1}, c_{k}\right),
$$

with equality occurring if and only if all $c_{k}$ lie along a (smooth) minimizing geodesic curve from $c_{0}$ to $c_{K}$ and $\operatorname{dist}\left(c_{k-1}, c_{k}\right)=\operatorname{dist}\left(c_{k}, c_{k+1}\right)$ for all $0<k<K$. Our approximation $\operatorname{dist}^{2}\left(\mathcal{M}^{\delta}, \phi_{\tau}\left(\mathcal{M}^{\delta}\right)\right) \approx \mathcal{W}\left[\mathcal{M}^{\delta}, \phi_{\tau}\left(\mathcal{M}^{\delta}\right)\right]$ turns the right hand side of (6) into our discrete path energy.

\section{Deformation energy}

Since we view shells as thin material layers, viscous friction is predominantly caused by two mechanisms (cf. Fig. 3):

(i) friction due to in-layer (tangential) shear or compression, (ii) friction due to (transversal) shear caused by bending.
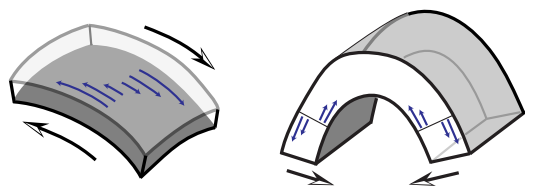

Figure 3: Tangential (left) and bending (right) distortion, leading to viscous friction.

Based on a suitable decomposition and approximation of the deformation energy in (3), (i) can be described in terms of membrane strains of $\mathcal{M}$, while (ii) is associated with bending strains of $\mathcal{M}$. This view can be made precise by relying on a well established theory for the approximation of elastic deformation energies and the geometry thereof. Indeed, (i) and (ii) are intimately related to the first and second fundamental forms of surfaces, respectively.

According to the path energy (4), it suffices to spell out elastic energies for pairs of surfaces $\overline{\mathcal{M}}$ and $\mathcal{M}$. Here we view $\overline{\mathcal{M}}$ and $\mathcal{M}$ as the undeformed and deformed surface (of zero thickness), respectively.

Membrane energy. The change of first fundamental forms induced by a deformation $\phi: \overline{\mathcal{M}} \rightarrow \mathcal{M}=\phi(\overline{\mathcal{M}})$ can be captured by a field $A[\phi]$ of symmetric positive definite linear mappings acting on tangent vectors of $\overline{\mathcal{M}}$, defined via

$$
\overline{\mathrm{I}}(A[\phi] v, w)=\mathrm{I}(d \phi(v), d \phi(w)) .
$$

Here $\bar{I}$ and I are the first fundamental forms on $\overline{\mathcal{M}}$ and $\mathcal{M}$, respectively, and $v, w$ are tangent vectors on $\overline{\mathcal{M}}$. Note that the corresponding quadratic form $\mathrm{I}^{*}(v, w)=\overline{\mathrm{I}}(A[\phi] v, w)$ is the pullback of the first fundamental form of $\mathcal{M}$ to $\overline{\mathcal{M}}$. Below, we provide an analogous construction for the second fundamental form.

From the viewpoint of elasticity theory $A[\phi]$ is the Cauchy-Green strain tensor field measuring tangential distortion. In fact, LeDret and Raoult [LR95] have shown in the context of $\Gamma$-convergence that to leading order, the energy of a deformation $\phi: \overline{\mathcal{M}}^{\delta} \rightarrow \phi\left(\overline{\mathcal{M}}^{\delta}\right)$ scales linearly in the thickness parameter $\delta$ and after rescaling with $\frac{1}{\delta}$ is given by the membrane energy

$$
\mathcal{W}_{\text {mem }}[\overline{\mathcal{M}}, \phi(\overline{\mathcal{M}})]=\int_{\overline{\mathcal{M}}} W_{\text {mem }}(A[\phi]) \mathrm{d} a,
$$

where the energy density $W_{\text {mem }}$ depends on the field $A[\phi]$. Inspired by the results in [WBRS11], we define the requisite energy density as

$$
W_{\text {mem }}(A)=\frac{\mu}{2} \operatorname{tr} A+\frac{\lambda}{4} \operatorname{det} A-\frac{2 \mu+\lambda}{4} \log \operatorname{det} A-\mu-\frac{\lambda}{4}
$$

where $\lambda$ and $\mu$ are the Lamé constants of a tangential Newtonian dissipation measure and where $\operatorname{tr} A$ and $\operatorname{det} A \operatorname{denote}$ the trace and determinant of $A$ as an endomorphism on the tangent bundle of $\overline{\mathcal{M}}$. Notice that $\operatorname{det} A \operatorname{describes}$ area distortion, while $\operatorname{tr} A$ measures length distortion. The polyconvex function $W_{\text {mem }}(A)$ is rigid body motion invariant, and the identity is the minimizer. The $\log \operatorname{det} A$ term penalizes material compression, which in the discrete setting prevents degeneration of triangles.

Bending energy. Let $\overline{I I}$ and II denote the second fundamental forms of $\overline{\mathcal{M}}$ and $\mathcal{M}$, respectively. We pull back II to $\overline{\mathcal{M}}$ via

$$
\mathrm{II}^{*}[\phi](v, w):=\mathrm{II}(d \phi(v), d \phi(w)),
$$

and we define linear mappings $\bar{B}$ and $B$ corresponding to $\overline{\mathrm{II}}$ and II $^{*}$, respectively. In detail, $\bar{B}$ is the shape operator on $\overline{\mathcal{M}}$, i.e. $\overline{\mathrm{I}}(\bar{B} v, w)=\overline{\mathrm{I}}(v, w)$, and $B$ is the pulled-back shape operator on $\mathcal{M}$, i.e. $\overline{\mathrm{I}}(B v, w)=\mathrm{II}^{*}[\phi](v, w)$. The difference $Q[\phi]:=\bar{B}-B$ is a field of linear operators acting on tangent vectors of $\overline{\mathcal{M}}$, known as the relative Weingarten map. Friesecke et al. [FJM02,FJMM03] demonstrated that for isometric deformations $\phi$ (for which $\mathcal{W}_{\text {mem }}[\overline{\mathcal{M}}, \phi(\overline{\mathcal{M}})]=0$ ) the leading order term is cubic in the thickness $\delta$ and after rescaling with $\frac{1}{\delta^{3}}$ is given by the bending energy

$$
\mathcal{W}_{\text {bend }}[\overline{\mathcal{M}}, \phi(\overline{\mathcal{M}})]=\int_{\overline{\mathcal{M}}} W_{\text {bend }}(Q[\phi]) \mathrm{d} a .
$$

Although other choices are conceivable, we consider the simple choice $W_{\text {bend }}(Q)=\|Q\|^{2}$, where $\|$.$\| denotes the$ 

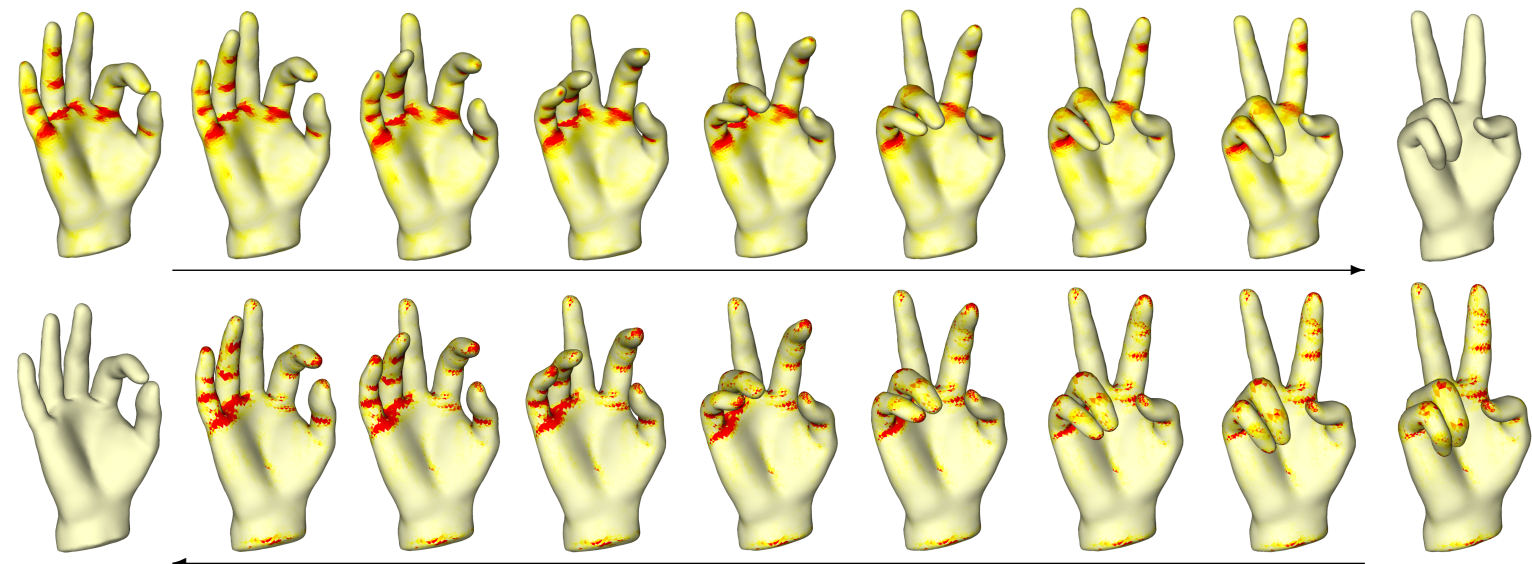

Figure 4: Discrete geodesic between two hand poses $A$ and $B$, where the top row shows results from computations of $A \rightarrow B$ and the bottom row shows (reversed) results from $B \rightarrow A\left(\right.$ both $\left.\eta=10^{-4}\right)$. The underlying dissipation is represented by the

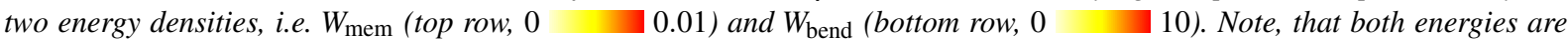
mainly concentrated at the joints, where dissipation due to bending is significantly larger than dissipation due to tangential distortion. The relative difference between path energies of the two sequences is $3 \%$. The deformation energy $\mathcal{W}_{\mathrm{def}}$ between two corresponding shapes in the two sequences is less than $0.01 \%$ of the deformation energy from the first to the final shell.

Frobenius norm. Notice that different from bending energies considered in graphics elsewhere, $W_{\text {bend }}(Q)$ takes into account the full change of second fundamental forms, not only the change of their traces (i.e., mean curvatures) - to the effect that changes of bending directions get accounted for appropriately.

Deformation energy. Combining membrane (7) and bending (9) contributions, we approximate the deformation energy (3) by

$$
\mathcal{W}[\mathcal{M}, \phi(\mathcal{M})]=\left(\mathcal{W}_{\text {mem }}+\eta \mathcal{W}_{\text {bend }}\right)[\mathcal{M}, \phi(\mathcal{M})]
$$

and rewrite the path energy (4) as

$$
\mathcal{E}\left[\mathcal{M}_{0}, \ldots, \mathcal{M}_{K}\right]:=\frac{1}{\tau} \sum_{k=1}^{K} \mathcal{W}\left[\mathcal{M}_{k-1}, \phi_{k}\left(\mathcal{M}_{k-1}\right)\right],
$$

which solely takes into account the two dimensional shell surfaces. Notice that according to the above discussion, $\eta=$ $\delta^{2}$; hence, $\sqrt{\eta}$ represents the thickness of the material.

Discussion. Figure 4 shows a discrete geodesic between two different hand poses. The underlying dissipation represented by the two energy densities is characterized by a strong concentration in regions of the joints, where dissipation due to bending is even more concentrated than dissipation due to tangential distortion. We note that geodesic paths need not be unique, and in particular buckling modes might lead to multiple (shortest) geodesic paths between two input shells (cf. Fig. 5). As discussed above, given two shells we regard the second as a deformed version of the first, i.e., the corresponding material of the second shell is just in a deformed configuration compared to its configuration in the first shell. Since every material point has a well-defined position, we view this deformation $\phi$ as a priori information when computing an optimal path between both shells. In the discrete setting we accommodate for this situation by working with meshes of a fixed connectivity, so that $\phi$ is indeed always well-defined.

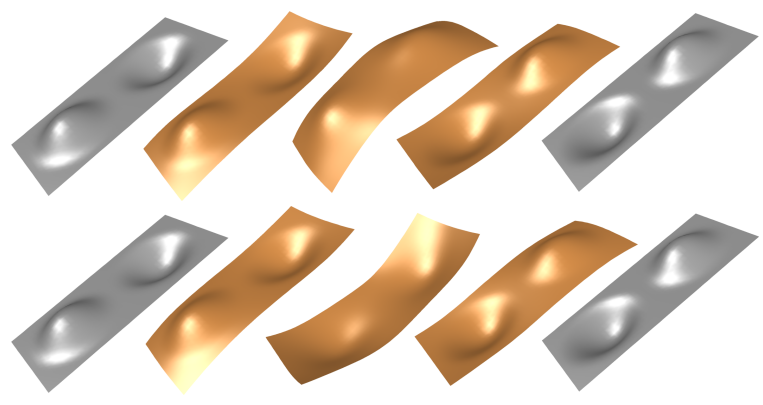

Figure 5: Symmetric setups can lead to non-unique geodesic paths. The flat plate as an intermediate shape is avoided since it would induce high tangential distortion. Therefore the bumps flip to the other side succesively, while the order of flipping does not effect the accumulated dissipation.

\section{Physical tuning of geodesic paths}

The above derivation of the deformation energy (11) completes our exposition of time-discrete geodesics for the case of smooth shells. Before introducing a corresponding version for discrete shells, we point out that our framework effortlessly allows a physically based tuning of "best" paths between shells. Our choice of physics-based deformation energies offers several advantages. Due to everyday experience, physical models come close to human intuition so that our framework tends to lead to intuitive discrete geodesic 

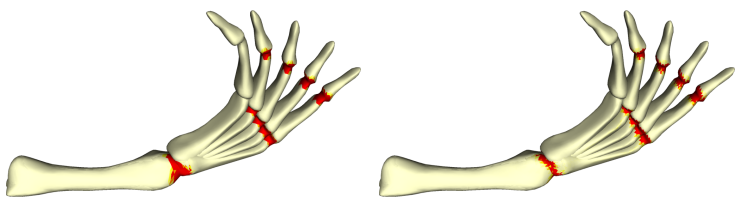

Figure 6: Discrete geodesic $\left(\eta=10^{-4}\right)$ between skeletons of a stretched and a bent finger (all shells superimposed), color-coded by membrane (left, $W_{\mathrm{mem}}, 0 \quad 10^{-4}$,) and bending energy (right, $W_{\text {bend, }} 0 \quad 10^{-1}$ ). Compare also to Fig. 5 in [KMP07].

paths. In particular, along a discrete path one can separate bending from stretching effects. The former especially play an important role for near-isometric deformations (see Fig. 6), an ubiquitous case in applications. Here, the energy dissipation due to bending serves as the proper path selection mechanism, without the use of additional (usually nonphysical) regularizers as in [KMP07]. In this respect, the parameter $\eta$ may also be seen as the natural regularization parameter (see Fig. 7). Obviously, the parameters $\eta, \lambda, \mu$ in

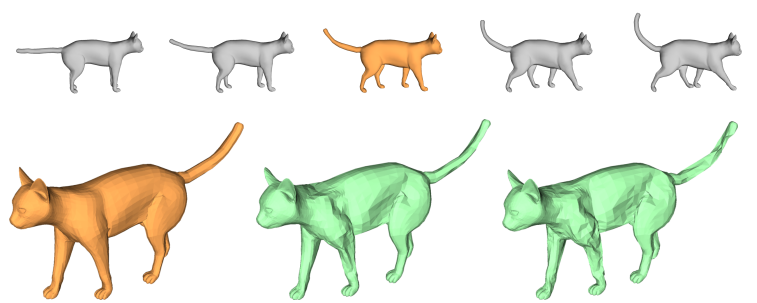

Figure 7: The bending term serves as a natural regularization; there is no need for artificial regularizers as in [KMP07] (top row, $\eta=0.8$ ). Decreasing $\eta$ leads to the occurance of creases until the underlying matching deformations are finally only as-isometric-as-possible (bottom, left to right: intermediate shape of a geodesic with $\eta=$ $0.8,0.01,0.0001)$.

our deformation energy have a physical interpretation and can be adjusted to change the typical behavior of shells. As discussed previously, $\eta$ describes the approximate squared width $\delta^{2}$ of the shell, a measure of resistance against bending: Geodesics with low $\eta$ allow for crumpling (Fig. 8) and if the initial and final shell are near-isometric, this prop-
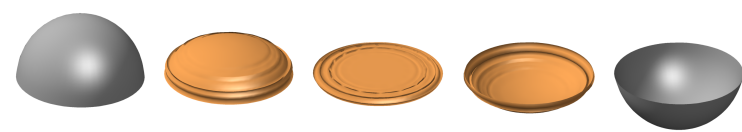

Figure 8: Discrete geodesic between two hemispheres with fixed rim. The low bending resistance allows crumpling $(\eta=$ $10^{-4}, \lambda=\mu=1$ ).

erty is approximately maintained for intermediate shells dur- ing the deformation. Vice-versa, a larger $\eta$ prevents crumpling at the cost of involving a fair amount of tangential stretching (Fig. 9,10). Both types of behavior are perfectly consistent with the intuitively optimal way to deform an aluminum foil or a thick metal sheet, respectively.

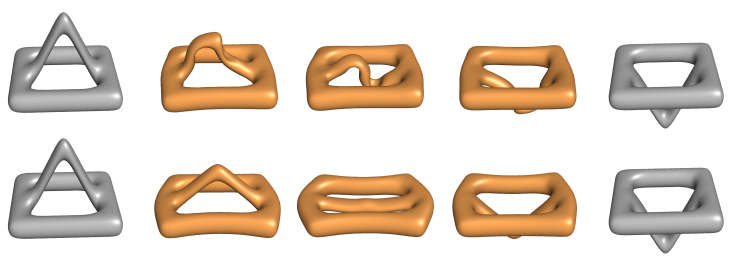

Figure 9: Discrete geodesic for low (top, $\eta=0.005)$ and high (bottom, $\eta=0.1)$ bending resistance $(\lambda=\mu=1)$. Stiff materials prefer the handle being folded (inducing bending dissipation) whereas soft materials stretch the outer frame (i.e. induce tangential distortion) to avoid folding.

The parameters $\lambda$ and $\mu$ represent the respective resistance against area and length changes, whose relative magnitudes also influence the appearance of discrete geodesics: In the previous examples we have pointed out that area compression can be avoided by out-of-plane buckling for sufficiently small bending resistance (see Fig. 11 bottom for an additional example). An alternative effect occurs for a low ratio $\mu / \lambda$ between shearing and dilation resistance, which allows for a simultaneous shell contraction in one and extension in the opposite direction (Fig. 11 top).
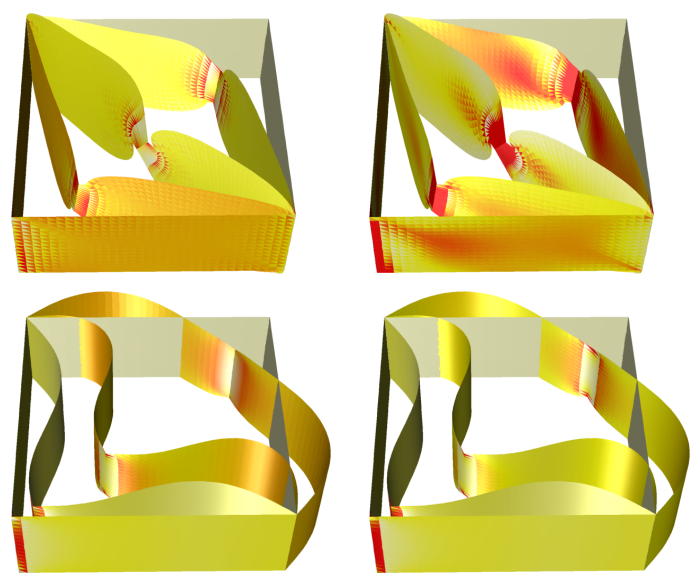

Figure 11: Discrete geodesics between two bracket shapes with fixed rim (all shapes superimposed), color-coded by $\operatorname{det} A[\phi]$ (left, $0 \quad 1.5$ ) and $\operatorname{tr} A[\phi]$ (right, $0 \quad 2.5$ ). While a high ratio $\mu / \lambda=20$ of shear to dilation resistance leads to the formation of loops (bottom), a low ratio of $\mu / \lambda=1 / 20$ leads to simultaneous extension and contraction in two orthogonal directions (top). Note that the central constriction of the intermediate shells is found as a means to reduce the bending resistance locally. 


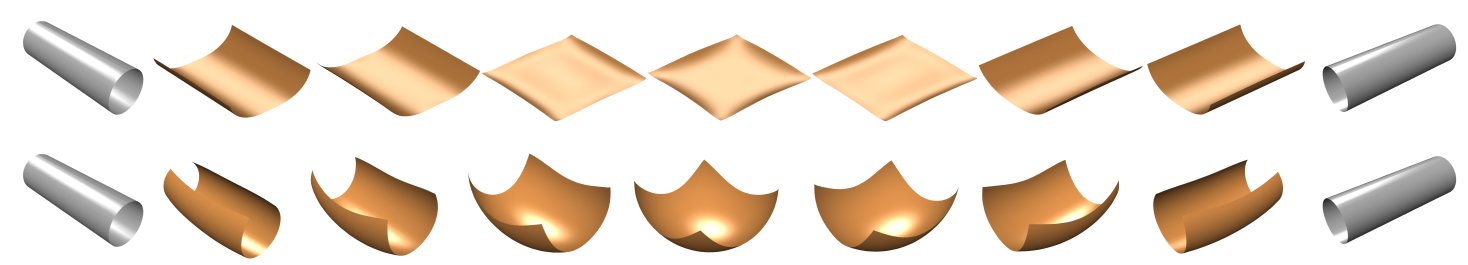

Figure 10: Discrete geodesic for low (top, $\left.\eta=10^{-6}\right)$ and high (bottom, $\eta=10^{-1}$ ) bending resistance $(\lambda=\mu=1)$.

\section{Discrete shells}

Mimicking our discussion of the smooth setting in Section 5, we require discrete versions of membrane and bending energies for pairs of polyhedral surfaces $\overline{\mathcal{M}}$ and $\mathcal{M}$ in order to compute the path energy (11) for sequences of polyhedral surfaces. As before, we view $\overline{\mathcal{M}}$ as the undeformed and $\mathcal{M}$ as the deformed two-dimensional surface. We also assume that $\overline{\mathcal{M}}$ and $\mathcal{M}$ have the same connectivity, to the effect that a piecewise linear mapping $\phi$ between these surfaces is readily available. Following our exposition in the smooth case, we require discrete notions of first and second fundamental forms, for which various versions can be found in the literature. Here we lay out our specific choices.

Membrane energy. We consider the standard constant strain triangle (CST) formulation to represent the membrane energy needed to deform a triangle $\bar{T}$ into a triangle $T$. In order to obtain compact expressions for the requisite first fundamental forms $\overline{\mathrm{I}}$ and I, notice that in dimension two, every quadratic form is uniquely determined by its action on three different vectors. Associating these vectors with the triangle edges $e_{1}, e_{2}, e_{3}$ of $T$, we define $\mathrm{I}_{i}:=\left\|e_{i}\right\|^{2}$ as the squared length of the $i$ th edge of triangle $T$. Analogous to the smooth setting, we define the pullback of the first fundamental form from $\mathcal{M}$ to $\overline{\mathcal{M}}$ by a piecewise linear mapping $A$ via $\overline{\mathrm{I}}(A \cdot, \cdot)=\mathrm{I}(d \phi \cdot, d \phi \cdot)$, which can be expressed per triangle by

$$
A_{\bar{T}}=\frac{1}{8|\bar{T}|^{2}} \sum_{i=1}^{3}\left(\mathrm{I}_{j}+\mathrm{I}_{k}-\mathrm{I}_{i}\right) \bar{t}_{i} \otimes \bar{t}_{i},
$$

where the indices $j=i+1(\bmod 3)$ and $k=i+2(\bmod 3)$ refer to the cyclic ordering of edges of $\bar{T}, \otimes$ denotes the outer product, $\bar{t}_{i}$ is the result of clockwise rotating edge $\bar{e}_{i}$ by $\pi / 2$ in the plane of $\bar{T}$, and $|\bar{T}|$ denotes the area of $\bar{T}$. The associated membrane energy takes the form

$$
\mathcal{W}_{\text {mem }}[\overline{\mathcal{M}}, \mathcal{M}]=\sum_{\bar{T}} W_{\operatorname{mem}}\left(A_{\bar{T}}\right)|\bar{T}|,
$$

where we sum over all triangles $\bar{T}$ of $\overline{\mathcal{M}}$ and we use the exact same energy density (8) as in the smooth case. However, notice that $A_{\bar{T}}$ has a null space corresponding to the normal direction; hence, we replace $\operatorname{det} A_{\bar{T}}$ by $\operatorname{det}\left(A_{\bar{T}}+\right.$ $\left.\frac{\left(\bar{e}_{1} \times \bar{e}_{2}\right) \otimes\left(\bar{e}_{1} \times \bar{e}_{2}\right)}{4|\bar{T}|^{2}}\right)$ in (8) in order to account for this effect in our implementation.
Bending energy. To be consistent with the above assumptions that first fundamental forms are constant per triangle, we work with second fundamental forms that have the same property. Since second fundamental forms are quadratic forms that account for the change of normals, we require three normals per triangle, which we associate with edge midpoints, by adopting and adapting ideas from [GGRZ06].

For every edge $e=T_{1} \cap T_{2}$ between two triangles, we define $N_{e}$ as the normalized sum of the unit normals belonging to the elements $T_{1}$ and $T_{2}$. For boundary edges, we take the respective triangle normal. With normals associated to edge midpoints, the (dis-

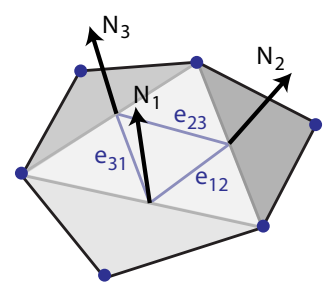
crete) 1 -form $d N$ acts on line segments connecting these midpoints. Indeed, fixing a triangle $T$ with edges $e_{1}, e_{2}, e_{3}$ and corresponding edge normals $N_{1}, N_{2}, N_{3}$ we find that $d N\left(e_{i j}\right)=\int_{e_{i j}} d N=N_{j}-N_{i}$, where $e_{i j}$ is the line segment connecting the midpoint of $e_{i}$ with that of $e_{j}$. Using the vector identity $e_{k}=-2 e_{i j}$, where $k$ is the complementary index to $i$ and $j$ in $T$, we accordingly define

$$
\mathrm{II}_{k}:=\mathrm{II}\left(e_{k}, e_{k}\right)=2\left(N_{i}-N_{j}\right) \cdot e_{k}
$$

as the action of the second fundamental associated with a triangle $T$ along the direction $e_{k}$. We make analogous definitions for the second fundamental form of the undeformed surface, i. e. , $\overline{\mathrm{II}}_{k}:=2\left(\bar{N}_{i}-\bar{N}_{j}\right) \cdot \bar{e}_{k}$.

Again, the quadratic forms $\overline{\mathrm{I}}_{\bar{T}}$ and $\mathrm{II}_{T}$ are uniquely determined by $\overline{\mathrm{II}}_{i}$ and $\mathrm{II}_{i}$, with $i \in\{1,2,3\}$, respectively. Analogous to the smooth setting, we define discrete shape operators via $\overline{\mathrm{I}}(\bar{B} \cdot, \cdot)=\overline{\mathrm{I}}(\cdot, \cdot)$ and $\overline{\mathrm{I}}(B \cdot, \cdot)=\mathrm{II}(d \phi \cdot, d \phi \cdot)$. Similar to our treatment of first fundamental forms, per triangle we obtain

$$
B_{\bar{T}}=\frac{1}{8|\bar{T}|^{2}} \sum_{i=1}^{3}\left(\mathrm{II}_{j}+\mathrm{II}_{k}-\mathrm{II}_{i}\right) \bar{t}_{i} \otimes \bar{t}_{i},
$$

and analogously for $\bar{B}_{\bar{T}}$ by replacing $\mathrm{II}_{i}$ by $\overline{\mathrm{I}}_{i}$ for $i \in$ $\{1,2,3\}$. As in the smooth case, we define a relative Weingarten map $Q_{\bar{T}}:=B_{\bar{T}}-\bar{B}_{\bar{T}}$. The respective bending energy 
is

$$
\mathcal{W}_{\text {bend }}[\overline{\mathcal{M}}, \mathcal{M}]=\sum_{\bar{T}} W_{\text {bend }}\left(Q_{\bar{T}}\right)|\bar{T}|,
$$

where again we use the exact same energy density $W_{\text {bend }}(Q)$ as in the smooth case, i.e. $W_{\text {bend }}(Q)=\|Q\|^{2}$.

Discussion. Our numerical experiments suggest that the resulting discrete geodesics are fairly mesh independent, even though the energy is only expected to become mesh independent for a very fine resolution. Figure 12 provides an example of this observation, where the path energy between a regular and an irregular discretization differs by $18 \%$ relative error, while the resulting shapes are fairly similar.

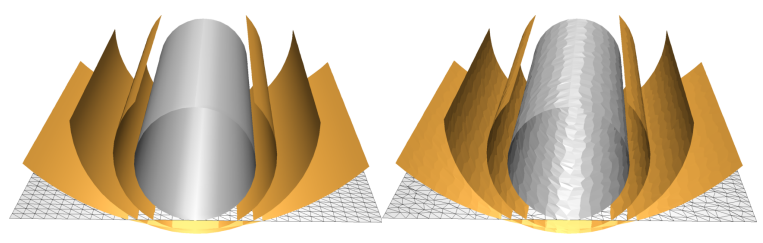

Figure 12: Discrete geodesic between flat and rolled state for regular (left) and irregular discretization (right), using 1100 nodes per shape $(\eta=0.001)$.

\section{Multilevel Optimization}

The minimization of the discrete deformation energy is a nonlinear optimization problem in $\mathbb{R}^{3 M(K-1)}$, where $M$ is the number of vertices of the triangulated shells, $K$ the number of time steps. We apply a Quasi-Newton method with DFP updating formula to approximate the Hessian, combined with a line search method subject to the strong Wolfe conditions. The stopping criterion is based on a threshold of $10^{-8} \mathrm{KM}$ in the squared $l^{2}$-norm for the energy gradient. Implementation details of the Quasi-Newton minimization are found in [NW99]; it only requires evaluation of the energy and its gradient. The formulas for the energy are provided in (10) and (11) with the requisite terms given by (13), (15). Note that (12) and (14) already provide matrix representations of the corresponding linear operators so that they can be directly inserted into $W_{\text {mem }}$ and $W_{\text {bend }}$. Given these formulae, it is a simple but tedious exercise to compute the gradient with respect to each vertex position.

Each energy and gradient evaluation has complexity $O(3 M K)$, where a parallelization over the $K$ discrete steps is straightforward. Table 1 shows average times for a single Quasi-Newton descent step.

Whenever feasible, the initialization is given by the linear interpolation of the two input shells $\mathcal{M}_{A}, \mathcal{M}_{B}$. Table 2 shows a convergence test for the simple test problem of a geodesic given by a family of expanding spheres with highly resolved triangulations. We experimentally observe fast convergence and a small lack of symmetry if the two input

\begin{tabular}{|l|cccc|}
\hline data & Fig. 6 & Fig. 9 & Fig. 4 & Fig. 13 \\
\#nodes $M$ & 2000 & 5500 & 13000 & 40000 \\
time (s) & 0.25 & 1.5 & 2 & 5 \\
\hline
\end{tabular}

Table 1: Average time for a single Quasi-Newton iteration (contains one gradient evaluation and several energy evaluations due to line search), where the $K$ discrete steps are treated in parallel on $K$ processors with $2.2 \mathrm{GHz}$ each.

\begin{tabular}{|c|c|c|c|}
\hline $\mathrm{K}+1$ & $r=2 \rightarrow r=1$ & $r=1 \rightarrow r=2$ & rel. error \\
\hline 2 & 55.0227 & 58.6711 & ב.066 \\
\hline 3 & 51.5990 & 52.0550 & .009 \\
\hline 5 & 50.6144 & 50.6688 & .001 \\
\hline 9 & 50.3408 & 50.3480 & .000 \\
\hline
\end{tabular}

Table 2: Path energy of a discrete geodesic with length $K+$ 1. The second (third) column corresponds to the geodesic from a sphere of radius 2(1) to a sphere of radius 1 (2).

shapes are exchanged. Not only the path energies but also the intermediate shells are robust with respect to exchanging the end shells (unless the end shells are connected by multiple geodesics as in Figs. 5 or 11 so that the realized discrete path depends on the path initialization). Swapping initial and final shell in Fig. 4 results in visually almost identical intermediate shells which differ from the original ones only by a deformation with energy three orders of magnitude less than the energy of the deformation from the first to the final shell.

Similarly to [KMP07] we introduce a multilevel approach based on Progressive Meshes [HDD*93, Hop98]. The two input meshes $\mathcal{M}_{A}$ and $\mathcal{M}_{B}$ are simultaneously decimated using edge collapses while preserving a one-to-one mesh topology correspondence. In each decimation step a single edge is removed according to an appropriate error functional [GH97]. The geodesic optimization problem is first solved on the coarsest level. Then in a cascadic fashion the discrete geodesic is stepwise prolongated via nodal interpolation to the next finer grid level and optimized again.

Without a multilevel technique, the optimization in Fig. 6 (2000 nodes) needs about $3 \cdot 10^{5}$ iterations. The corresponding computation time of $2.5 \mathrm{~h}$ can be reduced to $628 \mathrm{~s}$ by using a hierarchy of four resolutions, where between each two levels the number of nodes reduces by the factor 0.3 (resulting in 1616, 4166, 15, and 2033 Quasi-Newton iterations from coarsest to finest level). However, we expect a further significant reduction in the number of iterations when replacing the Quasi-Newton method by a true Newton method.

Additionally we apply a temporal refinement process, i. e. we successively add intermediate shells. Every new shell is initialized via the calculation of a time-discrete geodesic of length 3 between the temporally neighboring shells. The spatial and temporal refinement are then combined leading to a multilevel optimization scheme in space-time as an effective method to compute discrete geodesic paths between complex input shells (cf. Fig. 13). 

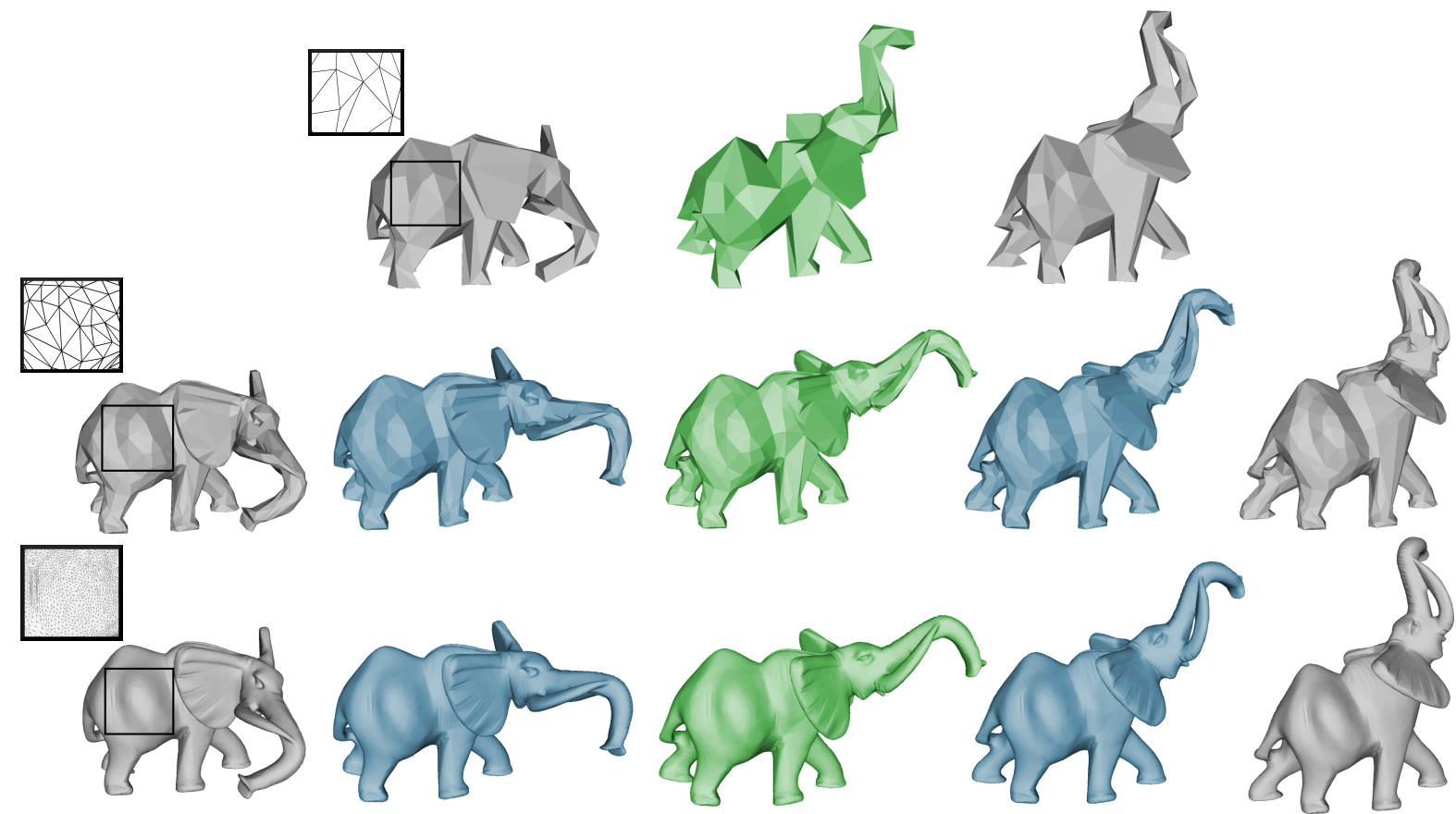

Figure 13: A sketch of the multilevel computation of a discrete geodesic, where progressive meshes and temporal refinement of the geodesic paths are applied with a reduction of nodes to less than $1 \%$ from bottom to top. Compare also to Fig. 1 in [KMP07] and Fig. 13 in [WDAH10].

Note that in the current algorithm we observe a lack of proper termination of the solver for large $\eta$ values $(\eta>1)$ representing a strong bending energy. Another numerical difficulty is caused by the fact that the mesh decimation algorithm in its current implementation does not take into account the local deformation strength, which varies across the shell. Thus, regions of strong deformation might get coarsened although they should better stay finely resolved. This might lead to slightly less balanced deformation steps at very coarse resolutions (cf. top green shell in Fig. 13). Possibly due to such effects, the needed total number of iterations strongly varies with the chosen number and resolutions of the different refinement levels, and heuristics for an optimal choice still have to be found.

\section{Conclusions}

We considered geodesic paths in the infinite-dimensional manifold of thin shell shapes as a model for smooth shape interpolation. The underlying metric is chosen as the physical energy dissipation due to shell stretching, shearing, and bending, where we interpret a shell variation as a deformation of a sheet of ductile material. Replacing the timecontinuous shell deformation by a finite sequence of stepwise deformations with associated elastic energies we arrive at an intuitive and simple definition of time-discretized geodesics as discrete paths which minimize the corresponding energies of the stepwise deformations. Our time dis- cretization allows quite coarse time resolution (cf. Tab. 2) and is a great advantage of the proposed model as it is algorithmically much simpler than the alternative of directly approximating solutions of the geodesic equations.

The elastic energy of the stepwise deformations splits into a physically sound hyperelastic membrane energy, which only measures the change in the first fundamental form, and a bending energy, which depends on the relative Weingarten map between undeformed and deformed shell and whose form can analytically be derived from three-dimensional elasticity theory. This strong physical foundation leads to very natural time-discrete geodesic paths, which can be tuned via a set of physical parameters. In particular the use of a physically correct bending energy already ensures smooth paths without any additional regularization.

For numerical computations, shells are triangulated, and the different energy contributions are approximated on the discretized shells. The derivation from a continuous formulation leads to the experimentally observed, valuable independence of the geodesic paths from the particular discretization and allows to employ a simple multilevel optimization approach.

Next steps necessarily involve the rigorous mathematical justification of the approach in the space continuous setting. Also, the same framework proposed here will be applied for geodesic extrapolation and parallel transport on the shell 
space. Furthermore, one might be interested in geodesic paths where the point relations between both end shapes are not prescribed.

Acknowledgements. End shapes for the discrete geodesics were kindly provided by Niloy Mitra (www.graphics.stanford.edu/ niloy/research/shape_space /shape_space_sig_07.html) and Olga Sorkine (graphics.csie.ncku.edu.tw/TF/). We thank Niloy Mitra for an interesting discussion on the regularization issue. Behrend Heeren and Benedikt Wirth were supported by the BMBF via CROPSENSe.net.

\section{References}

[AOW*08] Adams B., OvSJanikov M., Wand M., SeIDEL H.-P., GUIBAS L.: Meshless modeling of deformable shapes and their motion. In ACM SIGGRAPH/Eurographics Symposium on Computer Animation (Dublin, Ireland, 2008), ACM/Eurographics, Eurographics Association, pp. 77-86. 2

[BB11] BAUER M., BRUVERIS M.: A new Riemannian setting for surface registration. In Proceedings of the Mathematical Foundations in Computational Anatomy workshop (2011), Pennec X., Joshi S., Nielsen M., (Eds.), pp. 182-193. 2

[BBK09] BRonstein A. M., BRonstein M. M., KimMEL R.: Topology-invariant similarity of nonrigid shapes. International Journal of Computer Vision 81, 3 (2009), 281-301. 2

[CFK05] Charpiat G., Faugeras O., Keriven R.: Approximations of shape metrics and application to shape warping and empirical shape statistics. Foundations of Computational Mathematics 5, 1 (2005), 1-58. 2

[DGM98] Dupuis D., Grenander U., Miller M.: Variational problems on flows of diffeomorphisms for image matching. Quarterly of Applied Mathematics 56 (1998), 587-600. 2

[DHLM05] Desbrun M., Hirani A., Leok M., Marsden J.: Discrete exterior calculus, 2005. arXiv:math.DG/0508341 on arxiv.org. 2

[DKT08] Desbrun M., Kanso E., TONG Y.: Discrete differential forms for computational modeling. Oberwolfach Seminars: Discrete differential geometry 38 (2008), 287-324. 2

[EPT*07] Eckstein I., Pons J.-P., Tong Y., Kuo C.-C., DESBRUN M.: Generalized surface flows for mesh processing. In Eurographics Symposium on Geometry Processing (2007). 2

[FB11] FröHlich S., Botsch M.: Example-driven deformations based on discrete shells. Computer Graphics Forum 30, 8 (2011), 2246-âĂŞ2257. 2

[FJM01] FrIESECKE G., JAMES R. D., MÜLlER S.: Rigorous derivation of nonlinear plate theory and geometric rigidity. Tech. rep., Max-Planck-Institut, Leipzig, 2001. 2

[FJM02] Friesecke G., JAMES R. D., MÜLler S.: A theorem on geometric rigidity and the derivation of nonlinear plate theory from three dimensional elasticity. Communications on Pure and Applied Mathematics 55, 11 (2002), 1461-1506. 2, 4

[FJMM03] Friesecke G., James R., Mora M. G., Müller S.: Derivation of nonlinear bending theory for shells from threedimensional nonlinear elasticity by Gamma-convergence. C. $R$. Math. Acad. Sci. Paris 3368 (2003), 697-702. 2, 4

[FJSY09] FUChS M., JÜtTler B., SCherZER O., YANG H.: Shape metrics based on elastic deformations. J. Math. Imaging Vis. 35, 1 (2009), 86-102. 2
[FW06] FletCHER P., WhITAKER R.: Riemannian metrics on the space of solid shapes. In MICCAI 2006: Med Image Comput Comput Assist Interv. (2006). 2

[GGRZ06] Grinspun E., Gingold Y., REISMAN J., ZORIN D.: Computing discrete shape operators on general meshes. Eurographics 25 (2006). 2, 7

[GH97] GARLAND M., HeCKBERT P.: Surface simplification using quadric error metrics. ACM SIGGRAPH (1997), 209-216. 8

[GHDS03] Grinspun E., Hirani A. N., Desbrun M. SCHRÖDER P.: Discrete shells. In Eurographics/SIGGRAPH Syposium on Computer Animatio (2003). 2

[HDD*93] Hoppe H., DeRose T., Duchamp T., McDonald J., Stuetzle W.: Mesh optimization. Computer Graphics Proceedings, Annual Conference Series (1993). 8

[Hop98] Hoppe H.: Efficient implementation of progressive meshes. Computers and Graphics 22(1) (1998), 27-36. 8

[KKDS10] Kurtek S., Klassen E., Ding Z., SRivastava A.: A novel Riemannian framework for shape analysis of $3 \mathrm{~d}$ objects. In IEEE Computer Vision and Pattern Recognition (CVPR) (2010). 2

[KKG*11] Kurtek S., Klassen E., Gore J., Ding Z., SRIVASTAVA A.: Elastic geodesic paths in shape space of parametrized surfaces. IEEE Transactions on Pattern Analysis and Machine Intelligence to appear (2011). 2

[KMP07] Kilian M., Mitra N. J., Pottmann H.: Geometric modeling in shape space. In ACM Transactions on Graphics (2007), vol. 26, pp. \#64, 1-8. 2, 3, 6, 8, 9

[LR95] LeDret H., RaOult A.: The nonlinear membrane model as a variational limit of nonlinear three-dimensional elasticity. J. Math. Pures Appl. 73 (1995), 549-578. 4

[LSDM10] LIU X., SHI Y., DinOV I., Mio W.: A computational model of multidimensional shape. International Journal of Computer Vision Online First (2010). 2

[MM06] Michor P. W., MUMFORD D.: Riemannian geometries on spaces of plane curves. J. Eur. Math. Soc. 8 (2006), 1-48. 2

[NW99] Nocedal J., WRIGHT S. J.: Numerical Optimization. Springer, New York / Berlin, 1999. 8

[SKJJ11] SRIVASTaVa A., KLASSEn E., Joshi S. H., JERMyN I. H.: Shape analysis of elastic curves in euclidean spaces. Pattern Analysis and Machine Intelligence, IEEE Transactions on 33, 7 (july 2011), 1415-1428. 2

[SMSY11] Sundaramoorthi G., Mennucci A., Soatto S., YEZZI A.: A new geometric metric in the space of curves, and applications to tracking deforming objects by prediction and filtering. SIAM Journal on Imaging Sciences 4, 1 (2011), 109-145. 2

[Str45] Strutt J. W.: Theory of Sound, vol. 2. Dover Publications, 1945. 3

[SYM07] Sundaramoorthi G., YeZZI A., MennuCCI A.: Sobolev active contours. International Journal of Computer Vision. 73, 3 (2007), 345-366. 2

[WBRS11] WIRTH B., BAR L., RUMPF M., SAPIRO G.: A continuum mechanical approach to geodesics in shape space. IJCV 93, 3 (2011), 293-318. 2, 4

[WDAH10] Winkler T., Drieseberg J., Alexa M., HorMANN K.: Multi-scale geometry interpolation. Computer Graphics Forum 29, 2 (2010), 309-318. 2, 9 